\title{
Improved Embedding Efficiency and AWGN Robustness for SS Watermarks via Pre-Coding
}

\author{
H. Oktay Altun, Orhan Bulan, Gaurav Sharma, and Mark F. Bocko \\ University of Rochester, Rochester, NY, USA
}

\begin{abstract}
Spread spectrum (SS) modulation is utilized in many watermarking applications because it offers exceptional robustness against several attacks. The embedding rate-distortion performance of SS embedding however, is relatively weak compared to quantization index modulation (QIM). This limits the relative embedding rate of SS watermarks. In this paper, we illustrate that both the embedding efficiency, i.e. bits embedded per unit distortion and robustness against additive white gaussian noise (AWGN) can be improved by pre-coding of message followed by constellation adjustment on the SS detector to minimize the distortion on the cover image introduced by coded data. Our pre-coding method encodes $p$ bits as a $2^{p} \times 1$ binary vector with a single nonzero entry whose index indicates the value of the embedded bits. Our analysis show that the method improves embedding rate by approximately $p / 4$ without increasing embedding distortion or sacrificing robustness to AWGN attacks. Experimental evaluation of the method using a set theoretic embedding framework for the watermark insertion validates our analysis.
\end{abstract}

Keywords: E mbedding efficiency of robust watermarks, SS modulation, Pre-coding of watermark data.

\section{INTRODUCTION}

There are three important features characterizing a watermark design: robustness, embedding rate and fidelity of the watermarked image. These characteristics are competitive in nature and in order to improve one aspect, one may need to sacrifice performance in one or two of the other aspects. Depending on the specific needs of the application, the design can be more robust but have low embedding rate (e.g. ownership verification applications) or may offer high embedding rate with less distortion (e.g. in authentication applications). The embedding rate at a desired robustness level is limited by the watermark power available for embedding, which in turn is determined by the desired quality for the watermarked image. SS modulation ${ }^{1}$ is one example of a low embedding rate - high distortion method. A better rate - distortion performance can be achieved by QIM, ${ }^{2}$ usually at a cost of reduced robustness.

The limited power available for the watermark signal at a desired quality level of cover image may be more efficiently utilized by reducing the distortion per embedded bit. Efficiency improvements of this type are known for fragile LSB embedding, ${ }^{3,4}$ where the information to be embedded is coded into LSB bit plane prior to the LSB modification. Conventional LSB embedding with $K$ bits can require an embedding (Hamming) distortion as high as $\frac{K}{2}$ bits. Pre-coding using the dual of a $\left(2^{p}-1,2^{p}-p-1\right)$ binary Hamming code improves the embedding efficiency (distortion per embedded bit) by a factor $p$. The embedding rate offered by this code approach is however limited by the number of LSB bits in the image that can be utilized for the embedding: for a $512 \times 512$ image, utilizing the complete LSB plane the number of bits that can be embedded is approximately $\log _{2}(512 \cdot 512)=18$ with at most one bit change. However the scheme is more fragile compared to LSB embedding and change of any LSB bit in a block results in an error in the recovered data for the entire block of LSBs containing the modified LSB. Improvements in terms of embedding efficiency by utilizing linear codes and simplex codes for relatively longer messages have also been demonstrated. ${ }^{4}$

The embedding efficiency improvement in fragile settings is relatively easier compared to the robust schemes. This is due to the fact that the introduction of extra fragility is not critical in fragile schemes. Achieving a

Send correspondence to H.O. Altun: E-mail: altun@ece.rochester.edu, Telephone: 1585 275-2390, Address: Electrical and Computer Engineering Department, University of Rochester, Rochester, NY, 14627-0126, USA,

This work was supported by the Air Force Office of Scientific Research under grant number FA9550-07-1-0017.

Security, Forensics, Steganography, and Watermarking of Multimedia Contents $X$

edited by Edward J. Delp III, Ping Wah Wong, Jana Dittmann, Nasir D. Memon

Proc. of SPIE-IS\&T Electronic Imaging, SPIE Vol. 6819, 68191F, @ 2008 SPIE-IS\&T · 0277-786X/08/\$18 
similar improvement for robust watermark scenarios, on the other hand, is more challenging. This is mainly because the improved embedding efficiency often results in a decrease in embedding robustness. For example, we may consider reducing the embedding correlation threshold of SS watermarks. This will increase the number of bits embedded for the same distortion. However, the robustness will decrease proportionally as well.

In this paper, we present a method for improving embedding efficiency for SS watermarks without increasing the distortion or sacrificing robustness to AWGN attacks. This gain is achieved by pre-coding the data prior to embedding, utilizing a suitably modified signal constellation for the purpose of embedding and employing a maximum correlation detector at the receiver side. We consider both analytical and experimental evaluation of the proposed pre-coding method. In order to describe the embedding method in concrete terms, we specifically utilize the set theoretic embedding framework. ${ }^{5}$ The framework offers advantageous due to its capability to adapt to the visual content and its success in interference management between watermarks and cover image. It is however implicit that the precoding benefit may also be realized with alternate embedding methods. Despite the fact that the embedding efficiency can be improved without any sacrifice in terms of image quality or robustness to AWGN attacks in the proposed scheme, there is an increased computational burden due to the exponentially increasing number of sets*. We propose a set reduction method to reduce this cost at the embedder.

In Section 2 we briefly summarize the set theoretic embedding framework that we utilize for our experimental validation. The problem formulation in Section 2 also establishes notational conventions that we utilize subsequently. The proposed algorithm is presented in Section 3. In Section 4 we analyze the performance of the proposed algorithm and compare it with conventional spread spectrum in order to demonstrate its advantage. Results of experiments conducted in order to validate the proposed approach are presented in Section 5. Finally, concluding remarks are presented in Section 6.

\section{BACKGROUND}

\subsection{Overview of Set Theoretic Watermarking and POCS}

In set theoretic watermarking ${ }^{5,6}$ one represents each desired property of the watermarked image as a constraint set. A watermarked image is then obtained using an iterative algorithm that determines a point in the intersection of all the constraint sets. ${ }^{7}$ When the sets are convex the method of projection onto convex sets (POCS) provides a globally convergent algorithm for this purpose. Given $n$ convex sets $\left\{S_{i}\right\}_{i=1}^{n}$ the POCS method determines a point in their intersection by successive projections, defined mathematically by the iteration,

$$
f_{k+1}=\left(P_{S_{n}}\left(P_{S_{n-1}} \ldots P_{S_{1}}\left(f_{k}\right) \ldots\right)\right), \quad k=0,1, \ldots
$$

where $P_{S_{i}}$ is the projection operator onto set $S_{i}$ defined as $P_{S_{i}}(x)=\arg \min _{y \in S_{i}}\|y-x\|$. If the intersection of all the sets is non-empty, the sequence of images $\left\{f_{k}\right\}_{k=0}^{\infty}$ generated by POCS converges to a point in the intersection.

\subsection{Constraints for watermarking}

The common requirements of watermarking such as imperceptibility, robustness to compression, detectability and robustness to noise can be represented or approximated as convex sets. ${ }^{5,6}$ In this work, we consider only two of these sets. We denote the original image by $\mathbf{x}_{\mathbf{0}}$ and use $\mathbf{x}$ to indicate a candidate watermarked image. Watermarking requirements are then posed as constraints on $\mathbf{x}$. We also assume that the receiver detects the watermarks using pseudo noise(pn) sequences $\left\{\mathbf{w}_{j}\right\}_{j=1}^{K}$ for $K$ different watermarks, where the pn-sequences take values in $\{-1,+1\}$. These may be thought of as the $K$ spread spectrum watermarks though our framework does not explicitly embed these. We assume all images are $M \times N$ pixels so that all our vectors are $M N \times 1$ vectors.

Detectability and robustness to noise: At the receiver, the detector attempts to recover the embedded bits. Specifically for SS watermarks with the commonly used correlation detector, the correlation of the watermarked image with the key generated "watermark" pn-sequence indicates the received bit value. If the correlation is above a threshold $\tau_{d}$ a bit value of 1 is detected and if the correlation is below $\tau_{d}$ a bit value of 0 is detected. Typically $\tau_{d}=0$ due to symmetry ${ }^{8}$ and the detected bit value therefore corresponds to the sign of the correlation.

\footnotetext{
${ }^{*}$ The nature of these sets will become clearer in section 2
} 
We use the more general development here because we shall require it subsequently. The $K$ correlations with the $K$ different watermark sequences yield the $K$ different bits upon detection.

In order to embed the $K$ different bit values using the set theoretic watermarking framework, corresponding constraint sets $\left\{S_{1}{ }^{j}\right\}_{j=1}^{K}$ are defined within which the watermarked image is constrained to be

$$
\mathbb{S}_{1}^{j}= \begin{cases}\left\{\mathbf{x}: \mathbf{w}_{j}^{T}(\mathbf{x}-\overline{\mathbf{x}}) \leq \tau_{e}^{0}\right\}, & \text { if } b_{j}=0 \\ \left\{\mathbf{x}: \mathbf{w}_{j}^{T}(\mathbf{x}-\overline{\mathbf{x}}) \geq \tau_{e}^{1}\right\}, & \text { if } b_{j}=1\end{cases}
$$

where $\tau_{e}^{1}>\tau_{d}$ and $\tau_{e}^{0}<\tau_{d}$. The gaps $\left(\tau_{e}^{1}-\tau_{d}\right)$ and $\left(\tau_{d}-\tau_{e}^{1}\right)$ provide robustness in the detection of the embedded bits.

Imperceptibility of watermark: The watermark should adapt to the visual content of the cover image to maintain invisibility of the watermark noise. For this purpose, we employ constraints based on a masking model for visual perception $a^{5}$ :

$$
\mathbb{S}_{2} \equiv\left\{\mathbf{x}: \mathbf{l} \leq\left(\mathbf{x}-\mathbf{x}_{\mathbf{0}}\right) \leq \mathbf{u}\right\}
$$

where $\mathbf{l}$ and $\mathbf{u}$ are upper and lower bounds on pixel modifications that define the range of visually acceptable modification. These bounds are determined adaptively based on the original image $\mathbf{x}_{\mathbf{0}}$. The constraint exploits the fact that noise is less visible in textured regions compared to flat regions. A noise visibility function is modeled based on Voloshynovsky et al. ${ }^{9}$ and used to define a constraint set. ${ }^{5}$ Using the set definitions, a watermarked image meeting the constraints can be determined by using the POCS algorithm.

\section{PROPOSED ALGORITHM}

\subsection{Pre-coding of Data}

We illustrate the pre-coding of the data in Fig. 1. First we partition the message bits $c$ of length $L^{\dagger}$ into $\frac{L}{p}$ disjoint message blocks $c_{p}(i)$ where $i=1,2 \ldots, \frac{L}{p}$. Each message block has a length of $p$. Then we code each message block as a $2^{p} \times 1$ vector $\phi_{p}(i)$ which has a single 1 in the position whose index corresponds to the $p$-bit value for the message block.

Note that the simple index based pre-coding that is considered above may be thought of as a specific and simplified version of matrix embedding utilizing the dual binary Hamming codes. ${ }^{3}$ For matrix embedding Fridrich et al. ${ }^{4,10}$ have demonstrated significant improvement in embedding efficiency by utilizing more sophisticated codes for LSB embedding. Similar improvements of the method proposed here are worth investigating in follow-on research.

\subsection{Modified Constellation of $C S S$ and the Corresponding Detector}

We start by examining conventional spread spectrum $(C S S)$ constellation in the SS mark embedding context. The dual of energy in communication corresponds to distortion in watermarking settings. The origin represents the original cover signal and the squared distances of the constellation points from the origin represents the mean-squared distortion with respect to the original cover image.

The constellation of $C S S$ is symmetrically designed with respect to the origin as shown in Fig. 2(a) in order to minimize distortion when transmitted bit values 0 and 1 are equiprobable. However, the constellation of $C S S$ does not minimize the embedding distortion in the presence of pre-coding where it can be seen from Fig. 1 that the pre-coded data has a single one and $\left(2^{p}-1\right)$ zeros in a group of $2^{p}$ bits. There is a more efficient way to lay down an appropriate constellation that conforms to the intended coding. For this purpose, we perform an analysis to search for optimum constellation under pre-coding.

\footnotetext{
${ }^{\dagger} L$ is integer multiple of $p$.
} 


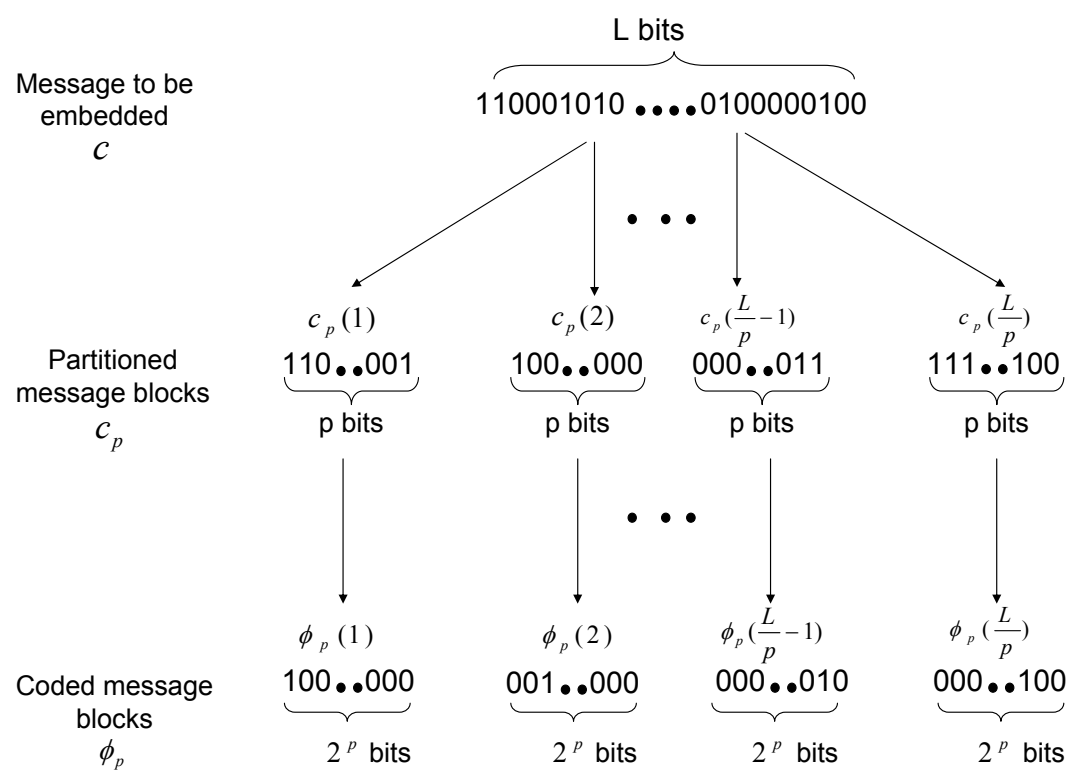

Figure 1. Illustration of coding scheme

To find the optimal constellation for this pre-coded data, we consider a shift of the constellation by $\Delta x$ as shown in Fig. 2(a). We keep the distance between the constellation points unchanged to maintain the same robustness. We then find the average distortion per embedded bit in the shifted constellation as follows:

$$
E(\Delta x)=\frac{2^{p}-1}{2^{p}}(-\sqrt{\varepsilon}+\Delta x)^{2}+(\sqrt{\varepsilon}+\Delta x)^{2} \frac{1}{2^{p}}
$$

where $\frac{2^{p}-1}{2^{p}}$ denotes the probability of embedding a bit value of 0 and $\frac{1}{2^{p}}$ denotes the probability of embedding a bit value of $1 .(-\sqrt{\varepsilon}+\Delta x)^{2}$ shows the distortion caused when a bit value of 0 is embedded and similarly $(\sqrt{\varepsilon}+\Delta x)^{2}$ shows the distortion caused by embedding a bit value of 1 . When we minimize the distortion $E(\Delta x)$ with respect to $\Delta x$, we find out the optimal shift as $\Delta x^{*}=\frac{2^{p}-2}{2^{p}} \sqrt{\varepsilon} . \Delta x^{*}$ approaches $\sqrt{\varepsilon}$ for high $p$ values. We need to shift the constellation by approximately $\sqrt{\varepsilon}$ to minimize the distortion for the pre-coded data-embedding. The practical implication is to code a bit value of 1 by the presence of the watermark and a bit value of 0 by its absence.

The corresponding detectors for $C S S$ and pre-coded data are shown in Fig. 2(b). In the $C S S$ detector $\tau_{e}$ denotes the embedding threshold when a bit value of 1 is embedded and $-\tau_{e}$ denotes the embedding threshold for a bit value of 0 . The natural detection threshold is 0 for CSS detector assuming transmitted bits are equiprobable. In the modified $C S S$ detector, $2 \tau_{e}$ is the embedding threshold for a bit value of 1 and the embedding threshold for a bit value of 0 is zero. The optimal detection threshold $\tau_{d}$ may not be necessarily in the middle of the embedding thresholds because bits are not equiprobable in the coded data. Finding the optimal $\tau_{d}$ is not necessary for our purposes in this paper because maximum correlation detector is employed in the detection rather than applying threshold detector. ${ }^{\ddagger}$

\subsection{Embedding the Coded Data}

We perform embedding by using the set theoretic framework as discussed in Sec. 2.1. The number of sets required for communication of $L$ bits is $\frac{L}{p} \times\left(2^{p}\right)$. We utilize these and the imperceptibility set and embed the data adaptively by successive projections until the convergence is met. Note that the number of constraints increases exponentially with increasing efficiency parameter $p$.

\footnotetext{
${ }^{\ddagger}$ Maximum correlation detector will be described in Sec. 3.4.
} 


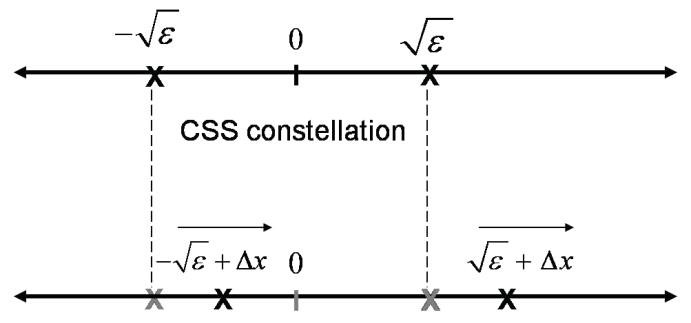

Shifted CSS constellation

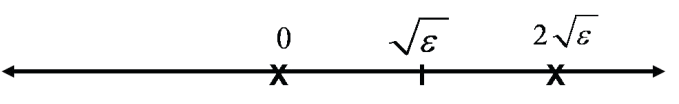

Modified CSS constellation

(a) $C S S$ constellation, shifted $C S S$ constellation and optimal constellation for the pre-coded data.
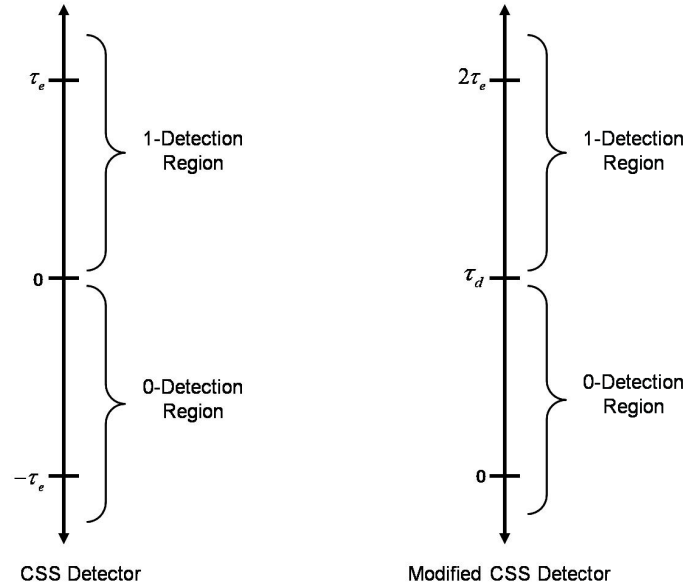

(b) Classical and modified SS detectors.

Figure 2.

The number of constraints for watermark embedding can be reduced to $\frac{L}{p}$ based on the observation that the bit 0 carrying pn-sequences are already uncorrelated with the image and imposing these sets $\mathbf{w}_{j}^{T}(\mathbf{x}-\overline{\mathbf{x}}) \leq \tau_{e}^{0}$ is therefore not required. We give the pseudocode for embedding process as follows:

1. Generate $2^{p}$ image sized, secret key controlled pn-sequences $w_{i}(j)$ for each coded message block, $j=$ $1,2, \ldots, 2^{p}$ and $i=1,2 \ldots, \frac{L}{p}$.

2. For each coded message block $\phi_{p}(i)$, find the pn-sequence corresponding to the bit value of 1 in the group $\S$

3. Project the image onto detectability sets corresponding to the pn-sequences mentioned at step 2 .

4. Project the image onto imperceptibility set. Check for convergence of the projection iterations. If converged, stop and output the current image (from projections) as the watermarked image, else go to step 3.

Figure 3 illustrates the embedding algorithm.

\subsection{Detection and Decoding}

The proposed algorithm with the pre-coded constellation adjusted SS $(P-C A S S)$ is well suited for employment of maximum correlation detection. Each coded message block includes single bit value of 1 and the detector can utilize the fact that there is single a bit value of 1 in each coded block. So the detector can simply check the maximum correlation in the received sequence to find the index location with the unity bit value. Employment of maximum correlation detector compensates the fragility introduced by set reduction at the embedder side. We illustrate the proposed detection and decoding with maximum correlation detector in Fig. 4 and give the pseudocode for detection and decoding process as follows:

1. Using the key shared with the embedder, generate the pn-sequence $w_{i}(j), j=1,2, \ldots, 2^{p}$ and $i=$ $1,2, \ldots, \frac{L}{p}$.

2. For each block i, calculate the correlations of the corresponding $2^{p}$ pn-sequences $\left\{w_{i}(j)\right\}_{j=1}^{2^{p}}$ with the received image.Find the maximum correlation for each coded block and decode the message for the $i^{t h}$ block as the index corresponding to the maximum correlation.

\footnotetext{
${ }^{\S}$ Actually, only these pn-sequences need to be generated at the embedder.
} 


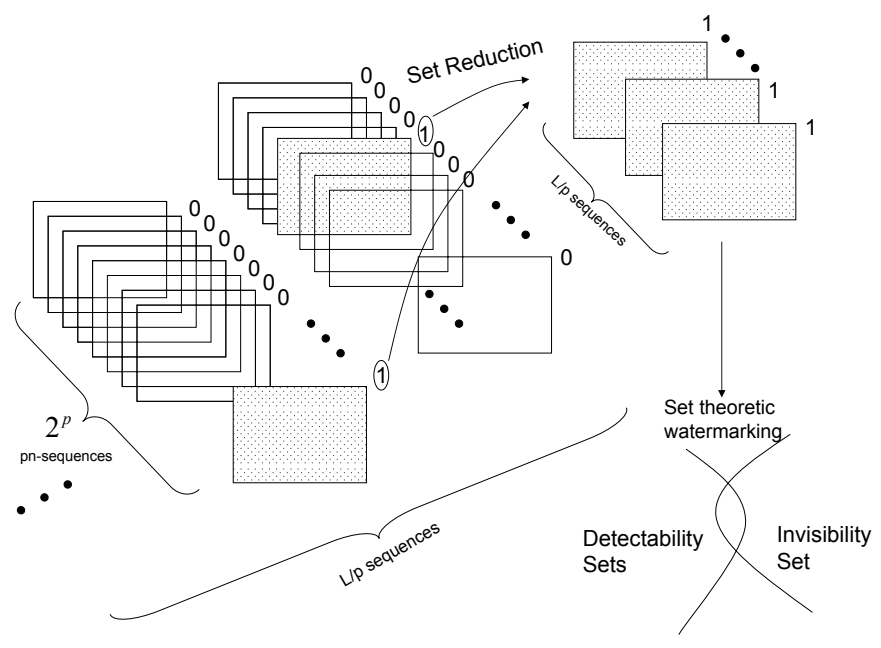

Figure 3. Proposed embedding scheme

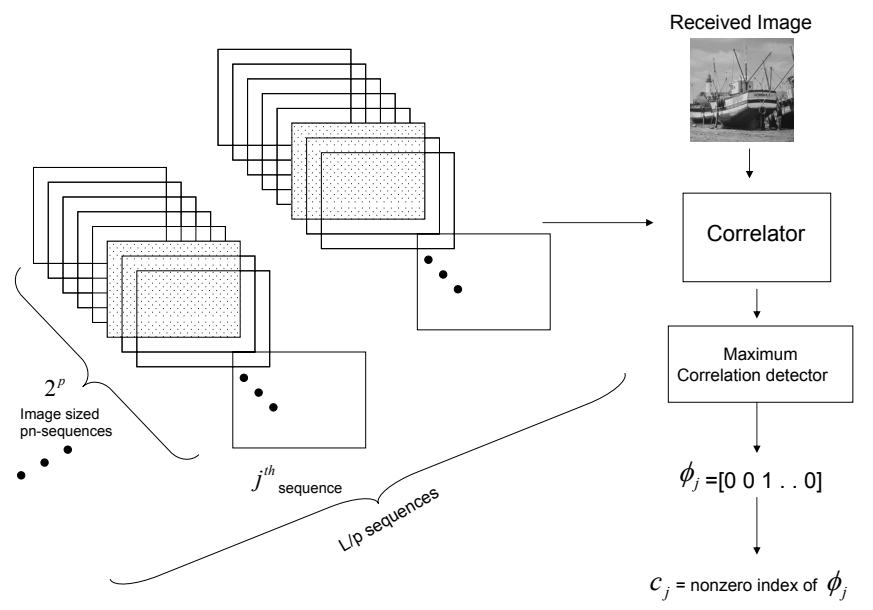

Figure 4. Proposed extracting scheme

\section{ANALYSIS}

In this section, we analytically investigate the performance of $C S S$ and $P-C A S S$ schemes under an AWGN channel. The measure for robustness is selected as probability of correctly decoding of a bit and the measure for distortion is chosen as distortion per embedded bit.

The received image is given by $\mathbf{x}_{r}=\mathbf{x}_{w}+\mathbf{n}$ where $\mathbf{n}$ is the channel noise modeled as an iid zero mean Gaussian vector with variance $\sigma_{n}^{2}$. Detectors for both $C S S$ and $P-C A S S$ compute correlations of the received image with pn sequences. These correlations are of the type $\mathbf{w}^{T} \mathbf{x}_{r}$, where $\mathbf{w}$ denotes the pn sequence. We assume these correlations are conditionally Gaussian random variables (given the embedded bit value) and we define two random variables to make the analysis clear. $X$ represents the correlation of received image with the pn-sequence when embedded bit is 0 . On the other hand, $Y$ stands for the correlation of pn-sequence with the received image when embedded bit is 1. We assume pdf's of $X$ and $Y$ can be approximated by the Gaussian distribution by invoking the central limit theorem (CLT).

\footnotetext{
"In fact $\mathrm{X}$ and $\mathrm{Y}$ represent the random variable corresponding to the computed correlation conditioned on whether
} 


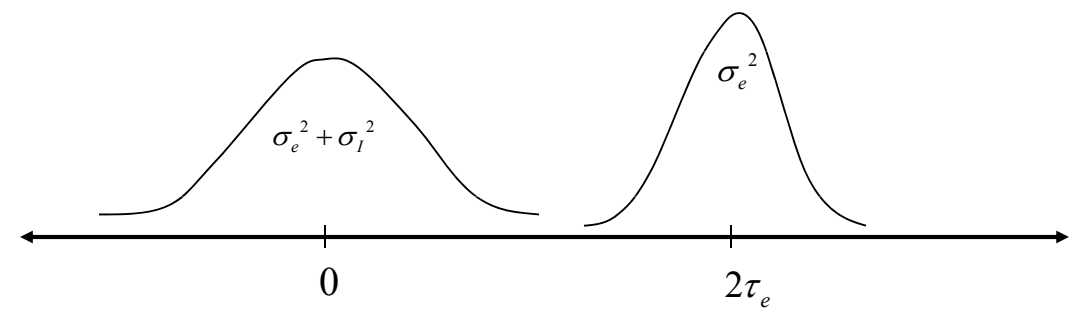

Figure 5. The noise distribution on a bit value of 0 and a bit value of 1 .

\subsection{Performance of $C S S$ Scheme}

In this case, $X$ and $Y$ are Gaussian random variables with identical variance $\sigma_{e}^{2}$ and mean values of $-\tau_{e}$ and $\tau_{e}$, respectively, where $\sigma_{e}^{2}=M N \sigma_{n}^{2}$.

\subsubsection{Robustness}

In this scheme, there is no coding prior to the embedding. We first modulate the message $c$ by spreading and embed into the cover. We calculate the bit error probability as follows:

$$
P_{e}=1-\frac{1}{2}\left[P\left(X<\tau_{d}\right)+P\left(Y>\tau_{d}\right)\right]=\frac{1}{2}\left[Q\left(\frac{\tau_{e}+\tau_{d}}{\sigma_{e}}\right)+Q\left(\frac{\tau_{e}-\tau_{d}}{\sigma_{e}}\right)\right]
$$

where $\tau_{e}=\tau_{e}^{1}=-\tau_{e}^{0}$ is the embedding threshold enforced by the embedding constraints (assumed to be symmetric). Since transmitted bits are equiprobable and the effect of noise is the same for a bit value of 0 and a bit value of $1, \tau_{d}$ is set to 0 as shown in Fig. 2(b).

\subsubsection{Distortion}

Upto a constant proportionality factor" ${ }^{\|}$, the average distortion per embedding is $\varepsilon$ as shown in Fig. 2(a). The expected overall distortion in the $C S S$ scenario is $\sum_{i=1}^{L} \varepsilon \cdot P(c(i)=1)+\varepsilon \cdot P(c(i)=0)$. Typically $P(c(i)=$ $1)=1 / 2$ reducing the expression to $L \cdot \varepsilon$. The distortion per embedded bit is therefore equal to $\varepsilon$.

\subsection{Performance of $P-C A S S$}

In this case, $X$ and $Y$ are Gaussian random variables with mean values of 0 and $2 \tau_{e}$ and variances $\sigma_{e}^{2}+\sigma_{I}^{2}$ and $\sigma_{e}^{2}$, respectively, where $\sigma_{e}^{2}=M N \sigma_{n}^{2}$. Figure 5 schematically illustrates the probability density function of the two random variables at the $P-C A S S$ detector.

\subsubsection{Robustness}

A similar robustness analysis can be performed for $P-C A S S$ case. In $P-C A S S$ scheme we partition the message $c$ to be embedded into $L / p$ blocks where $L$ is chosen to be a multiple of $p$. Each partitioned block $c_{p}(i)$ for $i=1,2 \ldots, L / p$ is pre-coded. These coded blocks are represented as $\phi_{p}(j)$ for $j=1,2 \ldots, L / p$. The probability of bit error for $P-C A S S$ is bounded above by the probability of block error. Therefore, we consider the block error probability in our analysis of $P-C A S S$ scheme, with the implicit understanding that the resulting expression is infact an upper bound on the probability of error.

the embedded bit is a zero or one, respectively.

"Determining this proportionality constant is not necessary for our purposes here. 
The probability of block error can be expressed as follows:

$$
\begin{aligned}
P_{e} & =1-P\left(\hat{\phi}_{p}(j)=\phi_{p}(j)\right) \\
& =1-\int_{-\infty}^{+\infty} P\left(Y>X_{1} \mid y\right) P\left(Y>X_{2} \mid y\right) \cdots P\left(Y>X_{2^{p}-1} \mid y\right) P(y) d y \\
& =1-\int_{-\infty}^{+\infty}\left(1-Q\left(\frac{y}{\sqrt{\sigma_{I}^{2}+\sigma_{e}^{2}}}\right)\right)^{2^{p}-1} \frac{1}{\sqrt{2 \pi \sigma_{e}^{2}}} \exp \left[\frac{-\left(y-2 \tau_{e}\right)^{2}}{2 \sigma_{e}^{2}}\right] d y
\end{aligned}
$$

This integral can be evaluated numerically. This expression also corresponds to the probability of correct detection for M-ary orthogonal modulation, ${ }^{11}$ to which our precoding scheme is equivalent.

\subsubsection{Distortion}

We embed a single bit value of 1 in each coded block. As shown in Fig. 1 there are $L / p$ blocks totally. Embedding bit value of zero does not cause any distortion on the cover image and the distortion caused by a bit value of 1 is $4 \varepsilon$ as shown in Fig. 2(a) (with an identical proportionality factor to that in Section 4.1.2.). So, overall expected distortion on the cover image becomes $\frac{L}{p} \cdot 4 \varepsilon$. Correspondingly the distortion per embedded bit is $\frac{4 \varepsilon}{p}$. Thus for $p \geq 4$ the proposed scheme has a lower distortion then CSS. The choice of constellations suggest that the schemes have similar robustness. The detection algorithms however, differ. We therefore compare robustness explicitly.

\subsection{Comparative Analysis}

We compare the performance of $C S S$ and $P-C A S S$ schemes. The robustness of each method depends on the parameters: $\tau_{e}, \sigma_{I}^{2}, \sigma_{e}^{2}$ and the message length $M N . p$ also plays a role for the $P-C A S S$ scheme. We choose $\tau_{e}=0.5, \sigma_{I}^{2}=0.004$. The value for $\sigma_{I}$ is determined empirically. So, we evaluate the scheme under AWGN attack with different attack powers (A.P), where attack power (A.P) in dB is calculated as: $10 \log _{10}\left(\sigma_{n}^{2}\right)$. All other parameters are kept constant for both schemes.

In Figs. 7 (a) to (c) we plot the probability of error as a function of $p$ for attack powers of 34, 37, and $40 \mathrm{~dB}$, respectively. Only values of $p \geq 4$ are included in the plots for which the distortion per embedded bit is lower for $P-C A S S$ than for $C S S$. Regions where $P-C A S S$ has a lower probability of error than $C S S$ therefore indicate operating points at which $P-C A S S$ has both a lower distortion and a higher robustness than CSS. For example, for an attack power of $37 \mathrm{~dB}$ the $P-C A S S$ scheme outperforms $C S S$ in terms of robustness for $p \leq 8$. In these operating regions, $P-C A S S$ offers higher robustness in addition to the embedding efficiency improvement of $p / 4$ over $C S S$. The graphic of Figure 6 illustrates the improvement achieved by $P-C A S S$.

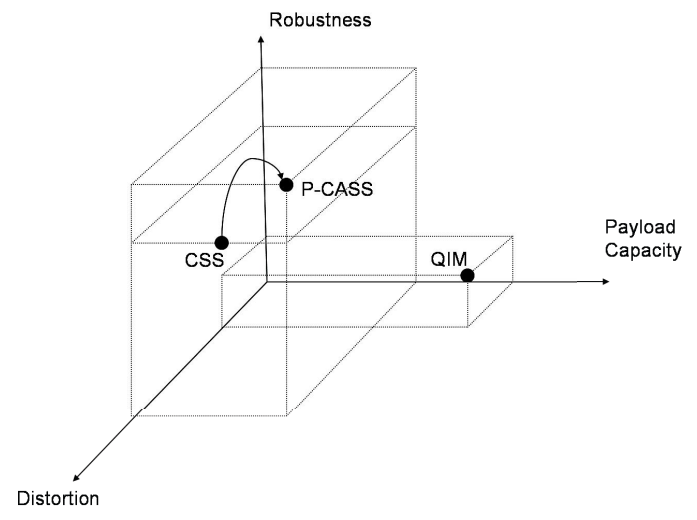

Figure 6. Illustration of the overall gain achieved by the proposed method. 


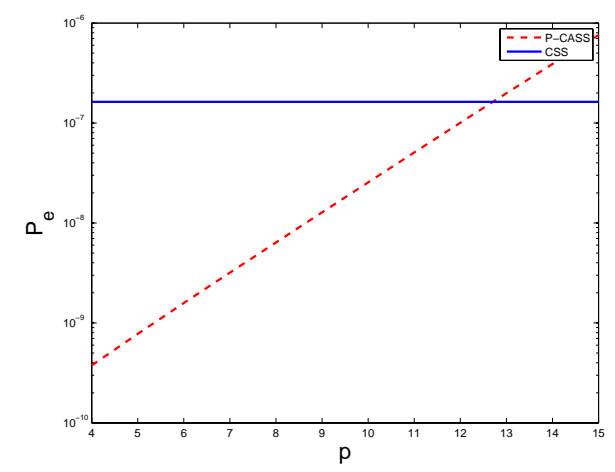

(a) $A \cdot P=34 \mathrm{~dB}$

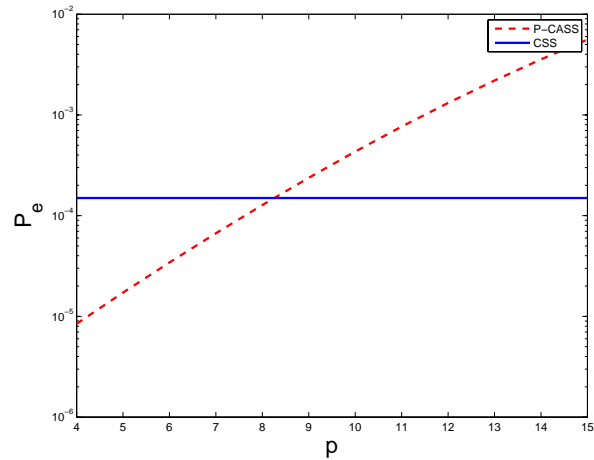

(b) $A \cdot P=37 \mathrm{~dB}$

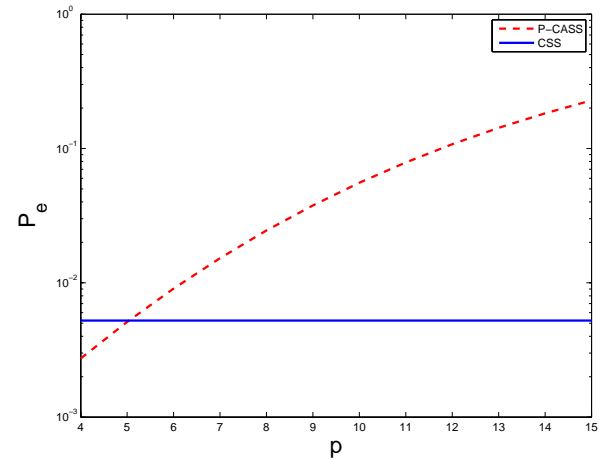

(c) $A . P=40 \mathrm{~dB}$

Figure 7. Comparative Analysis of $P-C A S S$ and $C S S$ under AWGN attacks with different attack powers. $P-C A S S$ scheme outperforms $C S S$ scheme up to some value of $p$ under different attack powers.

\section{EXPERIMENTAL RESULTS}

We experimentally evaluated the performance of the proposed scheme over 8 different $512 \times 512$ gray scale images from USC database. ${ }^{12}$ We adopt mean corrected correlation metric without normalization at the receiver side which provides robustness against additive noise and some resilience to valumetric scaling. ${ }^{8}$ The $C S S$ constellation is modified to reduce distortion and to allow the proposed precoding technique as discussed in Sec. 3.2.

We set $\tau_{e}=0.5 M N$ and $\tau_{d}=0$ as described in Sec. 3.2. We used $p=5$ for our pre-coding. For the texture masking model constraints in Eq. 3, we use the same parameters as in prior work $^{5}$

The analysis in Sec. 4 revealed that $P-C A S S$ has a distortion of $\frac{4 \varepsilon}{p}$ per embedded bit in comparison with $\varepsilon$ for $C S S$. In order to compare the methods under identical distortion, for $P-C A S S$ we use a data rate that is $p / 4$ times the data rate for $C S S$. Specifically, for our chosen parameter values, we consider the embedding of 200 bits for $C S S$ and of 250 bits for $P-C A S S$.

Table 1 compares the performance of $C S S$ and $P-C A S S$. The MSE distortion for the images is listed in the PSNR column of the Table 1, where the PSNR is calculated as: $P S N R=10 \log _{10}\left(\frac{255^{2}}{M S E}\right)$. We see in the table that the PSNR numbers for $C S S$ and $P-C A S S$ are quite close, indicating that our choice of suitable data rates does indeed result in (almost) equal distortion for the two methods as predicted by the analysis. Note that more bits are embedded for $P-C A S S$ than for $C S S$, implying an improved embedding efficiency for $P-C A S S$. At this point we want to make sure that we do not sacrifice robustness while improving the embedding efficiency. So we also check the robustness of these embedding techniques under severe AWGN attacks. The last two columns of the table demonstrate the bit error rates (BER) for these cases. We observe that under attack power $40 \mathrm{~dB}$ 
all bits are correctly decoded in both schemes. Although high robustness for CSS is typical, we observe that the higher robustness to AWGN is achieved when $P-C A S S$ scheme is employed.

Figure $8(\mathrm{c})$ illustrates the difference image between the watermarked Barbara image and the original image employing the proposed scheme. The watermark noise adapts nicely to the original image due to the interplay between the imperceptibility set and the detectability set during successive projections. Figures $8(\mathrm{a})$ and $8(\mathrm{~b})$ show the watermarked images with the conventional and proposed embedding schemes. As expected the perceptual quality of the images is similar. Figure $8(\mathrm{~d})$ reveals the severeness of the attacks we have introduced on images to test the robustness.

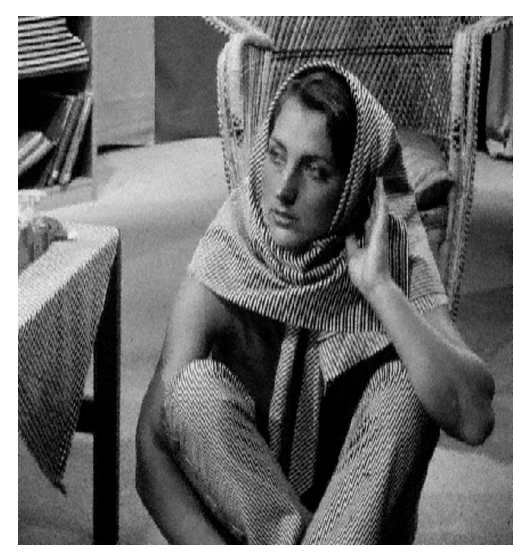

(a) Barbara image after 200 bits embedded with the $C S S$ scheme

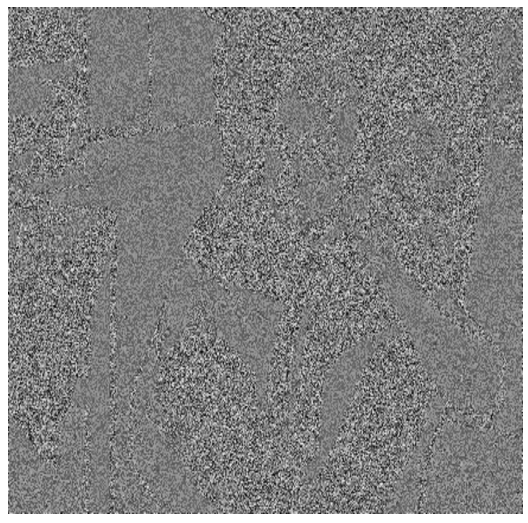

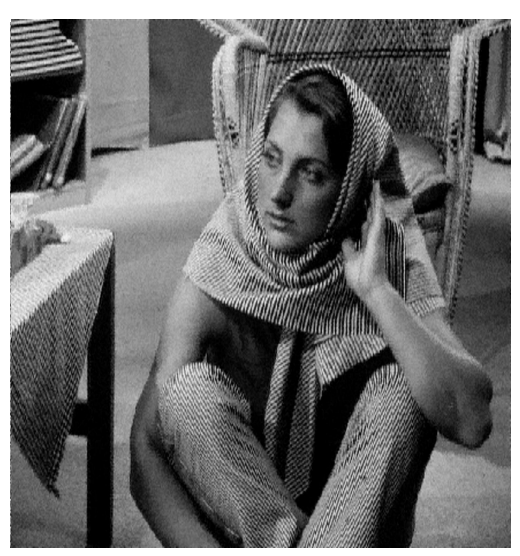

(b) Barbara image after 250 bits embedded with the $P-C A S S$ scheme (c) Difference image between watermarked and original Barbara images

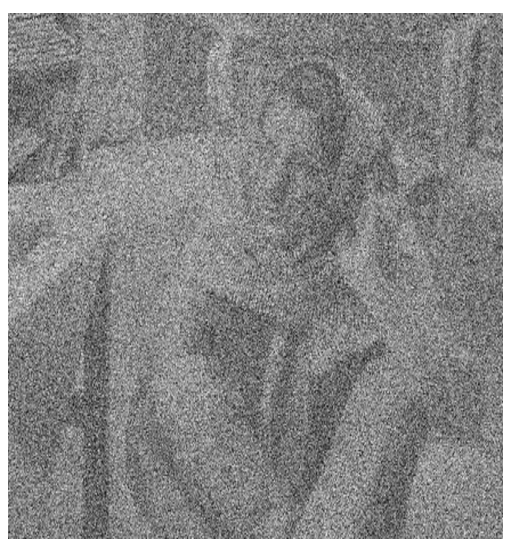

(d) Barbara image under AWGN attack with A.P $=40$ $\mathrm{dB}$ (Note that this is not PSNR)

Figure 8. 


\begin{tabular}{|c|l|c|c|c|cc|}
\hline Images & Method & Bits & $\begin{array}{c}\text { PSNR } \\
(\mathrm{dB})\end{array}$ & $\begin{array}{c}\text { SS watermarks } \\
\text { embedded }\end{array}$ & \multicolumn{2}{|c|}{ Bit error rate } \\
& & & A.P $(\mathrm{dB})=40$ & A.P $(\mathrm{dB})=41.76$ \\
\hline Barbara & $C S S$ & 200 & 30.46 & 200 & 0 & 0.01 \\
& $P-C A S S$ & 250 & 30.41 & 50 & 0 & 0 \\
\hline Goldhill & $C S S$ & 200 & 30.42 & 200 & 0 & 0.02 \\
& $P-C A S S$ & 250 & 30.67 & 50 & 0 & 0.012 \\
\hline Boat & $C S S$ & 200 & 29.92 & 200 & 0 & 0.03 \\
& $P-C A S S$ & 250 & 30.39 & 50 & 0 & 0.012 \\
\hline Zelda & $C S S$ & 200 & 30.77 & 200 & 0 & 0.025 \\
& $P-C A S S$ & 250 & 30.62 & 50 & 0 & 0.004 \\
\hline Washsat & $C S S$ & 200 & 30.78 & 200 & 0 & 0.015 \\
& $P-C A S S$ & 250 & 30.77 & 50 & 0 & 0 \\
\hline Lena & $C S S$ & 200 & 30.50 & 200 & 0 & 0.04 \\
& $P-C A S S$ & 250 & 30.53 & 50 & 0 & 0.024 \\
\hline Mandrill & $C S S$ & 200 & 30.96 & 200 & 0 & 0.015 \\
& $P-C A S S$ & 250 & 31.07 & 50 & 0 & 0 \\
\hline Peppers & $C S S$ & 200 & 30.54 & 200 & 0 & 0.035 \\
& $P-C A S S$ & 250 & 30.75 & 50 & 0 & 0.028 \\
\hline
\end{tabular}

Table 1. Embedding rates, distortion and robustness performance listed for both $C S S$ and $P-C A S S$ for 8 different images from USC image set.

\section{CONCLUSION}

In this paper, we proposed a pre-coding of watermark data coupled with adjustment in constellation of SS watermarks, in order to improve the embedding efficiency of SS watermarks. The proposed algorithm improves embedding efficiency of SS watermarks by $p / 4$ without any sacrifice of AWGN robustness. The maximum correlation detector also plays a critical role achieving this robustness. The computational burden introduced by coding can be reduced in the embedding side. We used a simple coding technique in this approach. More sophisticated coding techniques may improve the efficiency further, which can be a promising extension to this work.

\section{REFERENCES}

1. I. Cox, J.Kilian, F.T.Leighton, and T.Shamoon, "Secure spread spectrum watermarking for multimedia," IEEE Trans. Image Proc., vol. 6, no. 12, pp. 1673-1687, Dec. 1997.

2. B.Chen and G.W.Wornell, "Quantization index modulation: a class of provably good methods for digital watermarking and information embedding," IEEE Trans. Inform. Theory, vol. 47, pp. 1423-1443, 2001.

3. R. Crandall, "Some notes on steganography," Posted on Steganography Mailing List, http://os.inf.tudresden.de/ westfeld/crandall.pdf, 1998.

4. J. Fridrich and D. Soukal, "Matrix embedding for large payloads," IEEE Transactions on Information Security and Forensics, vol. 1, no. 3, pp. 390-394, 2006.

5. O. Altun, G. Sharma, M. Celik, and M. Bocko, "A set theoretic framework for watermarking and its application to semifragile tamper detection," IEEE Trans. Info. Forensics and Security, vol. 1, no. 4, pp. 479-492, December 2006.

6. O. Altun, G. Sharma, M. Celik, and M. Bocko, "Semifragile hierarchical watermarking in a set theoretic framework," in Proc. of ICIP, 2005, vol. 1, pp. 1001-1004.

7. P. L. Combettes, "The foundations of set theoretic estimation," Proceedings of the IEEE, vol. 81, no. 2, pp. 182-208, Feb. 1993.

8. I. J. Cox, M. L. Miller, and J. A. Bloom, "Digital watermarking," Morgan Kaufmann, Jul. 2001.

9. S. Voloshynoskiy, A. Herrigel, N. Baumgaertner, and T. Pun, "A stochastic approach to content adaptive digital image watermarking," Lecture Notes In Computer Science, pp. 211-236. 
10. J. Fridrich, M. Goljan, and D. Soukal, "Wet paper codes with improved embedding efficiency," Information Forensics and Security, IEEE Transactions on, vol. 1, no. 1, pp. 102-110, 2006.

11. John Proakis, Digital Communications, McGraw-Hill Science/Engineering/Math, August 2000.

12. Signal University of Southern California and Image Processing Institute., "The usc-sipi image database," http://sipi.usc.edu/services/database/Database.html,2001. 